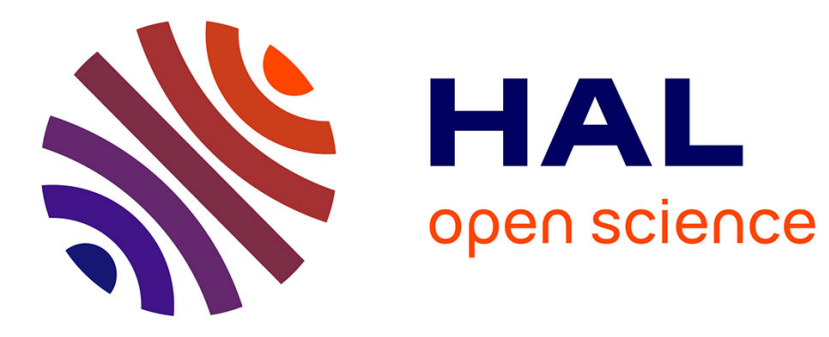

\title{
On the influence of initial state on gyrokinetic simulations
}

Guilhem Dif-Pradalier, P Grandgirard, P Sarazin, P Garbet, Philippe Ghendrih, P Angelino

\section{To cite this version:}

Guilhem Dif-Pradalier, P Grandgirard, P Sarazin, P Garbet, Philippe Ghendrih, et al.. On the influence of initial state on gyrokinetic simulations. Physics of Plasmas, 2008, 15, pp.42315 - 42315. 10.1063/1.2904901 . cea-01468363

\section{HAL Id: cea-01468363 https://hal-cea.archives-ouvertes.fr/cea-01468363}

Submitted on 15 Feb 2017

HAL is a multi-disciplinary open access archive for the deposit and dissemination of scientific research documents, whether they are published or not. The documents may come from teaching and research institutions in France or abroad, or from public or private research centers.
L'archive ouverte pluridisciplinaire HAL, est destinée au dépôt et à la diffusion de documents scientifiques de niveau recherche, publiés ou non, émanant des établissements d'enseignement et de recherche français ou étrangers, des laboratoires publics ou privés. 


\title{
On the influence of initial state on gyrokinetic simulations
}

\author{
G. Dif-Pradalier, V. Grandgirard, Y. Sarazin, X. Garbet, Ph. Ghendrih, and P. Angelino \\ Association Euratom-CEA, CEA/DSM/IRFM, CEA-Cadarache, F-13108 St. Paul-lez-Durance Cedex, France
}

(Received 11 January 2008; accepted 13 March 2008; published online 28 April 2008)

The influence of the initial state on the turbulence and transport is addressed in collisionless, global, and full-f $f$ gyrokinetic simulations solving both the equilibrium and the fluctuations. For two strongly differing initial states, it is found that the steady turbulent regime exhibits nearly identical statistical properties. This result is in marked contrast with the claim of different final states. In fact, a long transient with very different properties finally evolves towards the same turbulent regime for long simulation times. When the initial state is a local Maxwellian, i.e., constant on flux surfaces, a large-scale sheared electric potential develops on short time scales to compensate for the vertical curvature and grad-B drifts. We predict analytically (i) the temporal dynamics at short times of this electric potential, (ii) its poloidal structure, and (iii) its saturation time. All agree well with numerical simulations using the GYSELA code. The impact on the transport is twofold, as compared to the canonical initial state, where $f$ only depends on the motion invariants: (i) the turbulence is delayed due to a weaker effective growth rate, (ii) the same transport level is obtained at long times and the turbulence exhibits nearly identical statistical characteristics. In agreement, the electric potential of these two cases has the same magnitude despite very different transients.

(C) 2008 American Institute of Physics. [DOI: 10.1063/1.2904901]

\section{INTRODUCTION}

A reliable prediction of the turbulent transport is of utmost importance in fusion devices. It is known to strongly depend on the interplay between microturbulence and large scale flows. The major importance of a correct treatment of the zonal flows has been widely recognized. ${ }^{1}$ Direct numerical simulation using gyrokinetic modelling is now able to address such issues. In collisionless models, an important question has emerged on the choice of the initial state. Following Ref. 2, we will distinguish between the local and the canonical Maxwellian initial distribution functions. The latter, since it is a function of the motion invariants only, is an equilibrium solution of the gyrokinetic equation. Conversely, the former is not, and depends on the label of the flux surface.

When starting with a local Maxwellian, several authors, ${ }^{2-5}$ including us, reported on the spurious generation of large-scale sheared electric flows which hinder the turbulent modes and are likely to quench the onset of the turbulence. This paper will show that this result, correct at short simulation times (twice the linear growth, at most), breaks down in a fully developed steady turbulent state (at least five to ten times the linear growth). This state can be reached due to an improved coupling to two thermal baths located at the radial boundaries, and which provide the free energy for the system. In such a state, the statistical properties of the turbulence are independent of the choice of the initial state; no memory of its previous history is held within the system. More precisely, the transport level is the same since similar temperature profiles and turbulent heat flux are obtained. The turbulence itself also exhibits the same statistical properties, as, e.g., Fig. 8 will exemplify below.

On the other hand, when starting with a canonical Maxwellian, no such flows are generated and the turbulence de- velops first hand. It is acknowledged that a proper definition for an initial equilibrium state is a crucial piece of physics for gyrokinetic linear studies. Without flux-driven gyrokinetic simulations, it also avoids a detrimental delay since (i) long transients are avoided and (ii) profile relaxation in density and temperature is reduced. These two points are especially important for a new generation of global and full-f codes like the GYrokinetic SEmi-LAgrangian (GYSELA) code $^{6,7}$ since (i) large scale flows are intrinsically modelled and (ii) the mean profiles can evolve freely.

The main issues this paper will address are twofold: (i) how does the system evolve from its nonequilibrium initial state to an equilibrium and (ii) how does this evolution towards an equilibrium impact the onset of underlying instabilities, and ultimately the turbulent transport. The first point is crucial to discriminate between instability-driven fluctuations and time evolution towards the equilibrium. The second axis clarifies whether a nonequilibrium initial state can durably impact the dynamics of the turbulence itself. After a quick overview in Sec. II of the model we solve, we analytically predict in Sec. III the temporal dynamics, the poloidal structure, and the saturation time of the flows due to a nonequilibrium initial state. These results are successfully compared to dedicated numerical simulations with GYSELA. The saturation processes for these flows are discussed in Sec. IV and their nonlinear impact on the transport and the turbulence is reserved for Sec. V. A summary and discussion of the important results can be found in Sec. VI.

\section{A GYROKINETIC COLLISIONLESS MODEL FOR THE ITG INSTABILITY}

The model equations in GYSELA allow us to study collisionless turbulent transport driven by the ion temperature gradient (ITG) instability. In its present version, the turbu- 
lence is due to the fluctuations of the electrostatic potential $\phi$. The code is also written in a simplified toroidal geometry where magnetic surfaces are circular and concentric. The magnetic field writes $\mathbf{B}=B \mathbf{b}$, where $\mathbf{b}$ is the unit vector along the field lines, $\mathbf{b}=1 / \sqrt{1+(r / q R)^{2}}\left[\hat{\mathbf{e}}_{\varphi}+r /(q R) \hat{\mathbf{e}}_{\theta}\right], \quad \hat{\mathbf{e}}_{\varphi}$ and $\hat{\mathbf{e}}_{\theta}$ the unit vectors in the two periodic directions, respectively, toroidal and poloidal and $B=B_{0} R_{0} / R$. Quantities with a subscript 0 are computed at the magnetic axis $(r=0)$, $R=R_{0}+r \cos \theta$ refers to the major radius and $q(r)$ $=\mathbf{B} \cdot \nabla \varphi / \mathbf{B} \cdot \nabla \theta$ is the safety factor profile. The GYSELA code is based on a semi-Lagrangian scheme, ${ }^{6}$ applied to solve the gyrokinetic equation,

$$
\partial_{t} f+\left(\mathbf{v}_{E}+\mathbf{v}_{D}\right) \cdot \nabla f+v_{\|} \nabla_{\|} f+\mathrm{d}_{t} v_{\|} \partial_{v \|} f=0,
$$

where $\|$ refers to the direction of $\mathbf{b}, f$ is the gyroaveraged distribution function, and $\mathbf{v}_{E}$ is the electric drift velocity,

$$
\mathbf{v}_{E}=\frac{\mathbf{B} \times \nabla \bar{\phi}}{B^{2}} .
$$

Also, $\mathbf{v}_{D}$ refers to the curvature and grad-B drifts and in the low $\beta$ approximation reduces to

$$
\mathbf{v}_{D}=\frac{m v_{\|}^{2}+\mu B}{e B} \frac{\mathbf{B} \times \boldsymbol{\nabla} B}{B^{2}},
$$

where $m$ is the mass of the ions and $\mu=m v_{\perp}^{2} / 2 B$ is the adiabatic invariant. For simplicity, in the remainder of this paper, we will generically call $\mathbf{v}_{D}$ the curvature drift. The bar over $f$ or $\phi$ refers to the gyroaverage operation, $\bar{\phi} \equiv \mathcal{J} \cdot \phi$, where $\mathcal{J}$ is the gyroaverage operator,

$$
\mathcal{J} \cdot \phi=\int \mathrm{d}^{3} \mathbf{k}\left[\int_{0}^{2 \pi} \frac{\mathrm{d} \alpha}{2 \pi} e^{\imath \mathbf{k} \cdot \boldsymbol{\rho}_{s}}\right] \hat{\phi}_{\mathbf{k}} e^{\imath \mathbf{k} \cdot \mathbf{x}},
$$

where $\mathbf{x}$ is the position of the guiding centers, $\hat{\phi}_{\mathbf{k}}$ is the Fourier transform of $\phi, \rho_{s}$ is the ion gyroradius, and $\alpha$ is the gyration angle. Given that $\mathbf{k} \cdot \boldsymbol{\rho}_{s}=k_{\perp} \rho_{s} \cos \alpha$, the gyroaverage operator reduces in Fourier space to a multiplication by the Bessel function of first order $J_{0}\left(k_{\perp} \rho_{s}\right)$. In GyselA, it is replaced by its Padé approximation,

$$
J_{0}\left(k_{\perp} \rho_{s}\right) \approx \frac{1}{1+\left(\frac{k_{\perp} \rho_{s}}{2}\right)^{2}} .
$$

This is a crude approximation where sub-Larmor scales are strongly overdamped, as discussed in Ref. 8. However, it has the advantage of being valid when $k_{\perp} \rho_{s} \ll 1$ and keeps $J_{0}$ finite in the opposite limit $k_{\perp} \rho_{s} \rightarrow \infty$. Also, the evolution equation of the parallel velocity $\mathrm{d}_{t} v_{\|}$can be expressed as

$$
m \mathrm{~d}_{t} v_{\|}=-\mu \nabla_{\|} B-e \nabla_{\|} \bar{\phi}+\frac{m v_{\|}}{B} \mathbf{v}_{E} \cdot \nabla B,
$$

where the parallel gradient $\nabla_{\|}$reads

$$
\nabla_{\|}=\frac{1}{R}\left[\partial_{\varphi}+\frac{1}{q(r)} \partial_{\theta}\right] .
$$

It is worth noticing that Eq. (6), up to the first order in $\rho_{\star}=\rho_{s} / a$, can be recast into

$$
m \mathrm{~d}_{t} v_{\|}=-(\mu \boldsymbol{\nabla} B+e \boldsymbol{\nabla} \bar{\phi}) \cdot \frac{\mathbf{B}^{\star}}{\mathrm{B}^{\star}},
$$

where we have introduced the small tokamak parameter $\rho_{\star}, a$ the tokamak minor radius, and $\mathbf{B}^{\star}$ the classical modified magnetic field, $\mathbf{B}^{\star} \equiv \mathbf{B}+\left(m v_{\|} / e\right) \nabla \times \mathbf{b}$ whose norm reads $B^{\star}=B+\left(m v_{\|} / e\right) \mathbf{b} \cdot \nabla \times \mathbf{b}$. Up to this limit, the whole model equations have been checked to be fully consistent with the general gyrokinetic theory. ${ }^{9}$ Self-consistency is achieved with use of the quasineutrality equation,

$$
\begin{gathered}
\frac{e}{T_{e}(r)}\left[\phi-\phi_{00}\right]-\frac{1}{n_{\mathrm{eq}}(r)} \nabla_{\perp} \cdot\left[\frac{n_{\mathrm{eq}}(r)}{B_{0} \omega_{c i}} \nabla_{\perp} \phi\right] \\
=\frac{2 \pi B}{m n_{\mathrm{eq}}(r)} \int_{0}^{\infty} d \mu \int_{-\infty}^{+\infty} d v_{\|} \mathcal{J} \cdot\left[f-f_{\text {init }}\right],
\end{gathered}
$$

where $\phi_{00} \equiv \iint d \theta d \varphi / 4 \pi^{2} \phi$ accounts for the magnetic fluxsurface average. The electronic response is adiabatic, $\delta n_{e} / n_{\mathrm{eq}}=e\left(\phi-\phi_{00}\right) / T_{e}(r)$. The ion cyclotron pulsation is $\omega_{c}$, $f_{\text {init }}$ refers to the initial gyroaveraged distribution function for the ions and initially, the electric potential $\phi$ is a perturbation. The Laplacian in the left-hand side comes from the Taylor expansion of the double gyroaveraged operator ${ }^{10}$ and validly describes a quasineutral plasma in the $k_{\perp} \rho_{s} \lesssim 1$ limit. Such an approximation is consistent with the Padé approximation of Eq. (5). Obviously, small scales should be treated more accurately when nonadiabatic electrons are included, either trapped or passing. The gyroaverage operator will be optimized in this perspective.

\section{SELF-GENERATED LARGE SCALE SHEARED ELECTRIC FLOWS DUE TO INITIAL CONDITIONS}

In this section, we concentrate on the dynamics of the plasma which occur when the initial distribution function $f_{\text {init }}$ is not an equilibrium solution of the gyrokinetic Eq. (1). The system is initialized with the local Maxwellian, Eq. (10), namely $f_{\text {init }} \equiv f_{\mathrm{LM}}$. We write

$$
\begin{aligned}
& f_{\mathrm{LM}}=\frac{n(r)}{[2 \pi T(r) / m]^{3 / 2}} e^{E / T(r),} \\
& f\left(r, \theta, v_{\|}, \mu, t\right)=f_{\mathrm{LM}}(r, E)+\delta f_{\mathrm{LM}}\left(r, \theta, v_{\|}, \mu, t\right),
\end{aligned}
$$

where $E$ stands for the total energy $m v_{\|}^{2} / 2+\mu B$ and the axisymmetry of the problem imposes $f$ to be independent of the toroidal angle. Initially, $\delta f_{\mathrm{LM}}(t=0)=0$. We call such a case out of equilibrium. Characterizing the dynamics of such a system essentially consists of determining the radial component of the electric field which builds up. At vanishing electric field, an equilibrium flow along the parallel direction must develop to balance the vertical polarization associated with the ion curvature drift. It is analogous to the PfirschSchlüter current carried by the electrons. With a local Maxwellian initial state which is even in $v_{\|}$, no such flow can exist initially or develop on short times. The remaining possibility for the system consists of generating a large-scale polarization flow in the poloidal plane which in turn can be seen as resulting from a large-scale driven electric potential. In what follows, we calculate this electric potential and give 
a prediction of the level, time growth, and saturation time of such flows.

In this framework, at short times, $\delta f_{\mathrm{LM}}$ is a perturbation. We restrict its Fourier expansion to the first modes only, which appear to be dominant,

$$
\delta f_{\mathrm{LM}} \approx f_{00}(r, E, t)+f_{10}^{s}(r, E, t) \sin \theta+f_{10}^{c}(r, E, t) \cos \theta,
$$

where $f_{00}$ corresponds to the axisymmetric mode, $(m, n)=(0,0), f_{10}^{s}$ and $f_{10}^{c}$ correspond to the sidebands, $(m, n)=( \pm 1,0), m$ and $n$ being, respectively, the poloidal and toroidal mode numbers. As far as analytical calculations are concerned, in this section, only $n=0$ modes are considered, which allows one to focus on the dynamics of equilibrium axisymmetric flows. Conversely, no such constraint is made on the numerical results where all possible $(n, m)$ modes are allowed to develop and interact. The very good agreement between the two approaches emphasizes the crucial role of the equilibrium $n=0$ modes. With $f_{\mathrm{LM}}$ as an initial state, the quasineutrality equation is satisfied for a vanishing electric potential $\phi(t=0)=0$. Therefore, initially at least, $\phi$ is a perturbation; we also expand it on its first Fourier modes,

$$
\phi(r, \theta, t) \approx \phi_{00}(r, t)+\phi_{10}^{s}(r, t) \sin \theta+\phi_{10}^{c}(r, t) \cos \theta .
$$

No ordering here is presupposed between, respectively, $f_{00}$, $f_{10}^{s}, f_{10}^{c}$, and $\phi_{00}, \phi_{10}^{s}, \phi_{10}^{c}$. The small parameter $\epsilon$ of this study is

$$
\epsilon=\max \left(\frac{f_{00}}{f_{\mathrm{LM}}}, \frac{f_{10}^{s}}{f_{\mathrm{LM}}}, \frac{f_{10}^{c}}{f_{\mathrm{LM}}}\right)
$$

which will be shown to scale like $\rho_{\star}$. The sidebands $f_{10}$ build up because of the polarization due to the curvature drift. It follows that at short times, Eq. (1) writes at leading order in $\epsilon$,

$$
\partial_{t} f+\mathbf{v}_{D} \cdot \nabla f=0 .
$$

As far as analytical calculations are concerned, in the remainder of this section, gyroaveraged quantities will be assumed to be equal to the quantities themselves, namely, $\mathcal{J} \equiv$ Identity. Such a simplification is valid in the limit of large gradient lengths with regard to $\rho_{s}$, as in the case of the present analysis. Equation (15), coupled to the quasineutrality equation (9), enable us to calculate the time evolution of both the perturbed distribution function and the electric potential. The detailed calculation can be found in Appendix A. It is possible to selectively derive the dynamics of each perturbed part, given its parity in the poloidal angle. The main equation on the distribution function which governs the dynamics of the system at short times is

$$
f_{10}^{s}=\frac{v_{d} f_{\mathrm{LM}}}{\mathcal{L}_{p}} t
$$

with $v_{d}=\left(m v_{\|}^{2}+\mu B\right) / e R_{0} B_{0}, T_{\mathrm{eq}} / e B_{0}=\rho_{s} c_{s}$ and $c_{s}^{2}=T_{\mathrm{eq}} / m$. For what follows, we note $L_{X}^{-1} \equiv \partial_{r} X / X$ and $\kappa_{X} \equiv \partial_{r r} X / X$. At last, $\mathcal{L}_{p}^{-1} \equiv L_{n}^{-1}+L_{T}^{-1}\left(E / T_{\mathrm{eq}}-3 / 2\right)$. When coupled to the quasineutrality equation, this latter equation, yields the time evolution of $\phi_{10}^{S}$ at first order in $\rho_{\star}$,

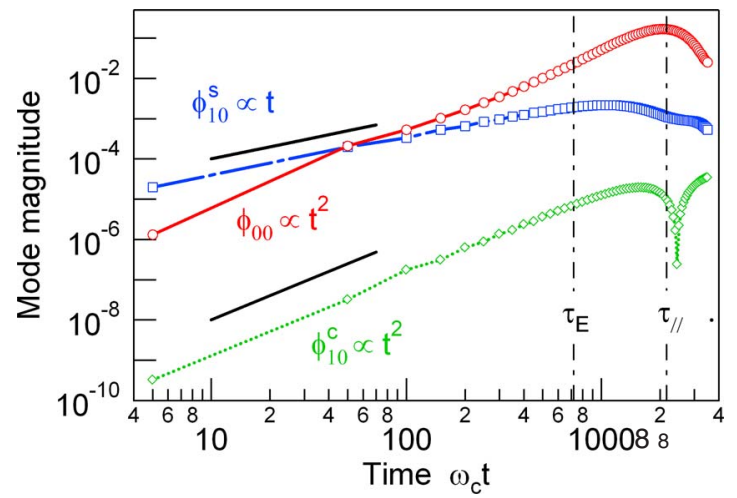

FIG. 1. (Color online) Time evolution of the transient electric potential when the plasma is initially out of equilibrium. (Solid line) analytical estimation of the growth rate; (dotted line) the complete simulation with $\operatorname{GySELA}\left(\rho_{\star}=1 / 400\right)$.

$$
\begin{aligned}
& \frac{e}{T_{\mathrm{eq}}} \phi_{10}^{s} \approx \Omega_{10}^{s} t, \\
& \Omega_{10}^{s}=\frac{2 \rho_{s} c_{s}}{R_{0} L_{p}} .
\end{aligned}
$$

The detailed calculation shows that Eq. (17) is the key equation which allows us to calculate the time evolution for all the other perturbed quantities. The vertical charge polarization due to the curvature drift is the driving effect which explains the growth of an electrostatic potential at short times. Coupling between $\phi_{10}^{s}$ and $f_{00}$ (resp. $f_{10}^{c}$ ) generates the axisymmetric (resp. cosine) component of the electric potential,

$$
\begin{aligned}
& \frac{e}{T_{\mathrm{eq}}} \phi_{10}^{c} \approx \Omega_{10}^{c} t^{2}, \\
& \Omega_{10}^{c}{ }^{2}=\frac{\left(\rho_{s} c_{s}\right)^{2}}{r R_{0} L_{p} L_{n}}, \\
& \frac{e}{T_{\mathrm{eq}}} \partial_{r r} \phi_{00} \approx \Omega_{00}^{2} t^{2}, \\
& \Omega_{00}^{2}=-\frac{7}{4}\left[\frac{1}{L_{n}}+\frac{4}{7 L_{p} L_{T}}+\frac{12}{7 L_{p}}\left(\frac{1}{L_{p}}+\frac{1}{r}\right)+\frac{16}{7} \kappa_{p}-\kappa_{n}\right] \\
& \quad \times\left(\frac{c_{s}}{R_{0}}\right)^{2} .
\end{aligned}
$$

Equations (17), (19), and (21) are predicted to characterize the dynamics of the system in response to the vertical polarization drift.

These analytical predictions can be checked with GYSELA. The code is initialized with the local Maxwellian and solves the full self-consistent problem, Eqs. (1) and (9). The temperature gradient is taken well below the ITG threshold to prevent the onset of any instability. The case above the threshold is detailed in Sec. V. Figure 1 compares the full numerical solution to the analytical predictions, Eqs. (17), (19), and (21). 


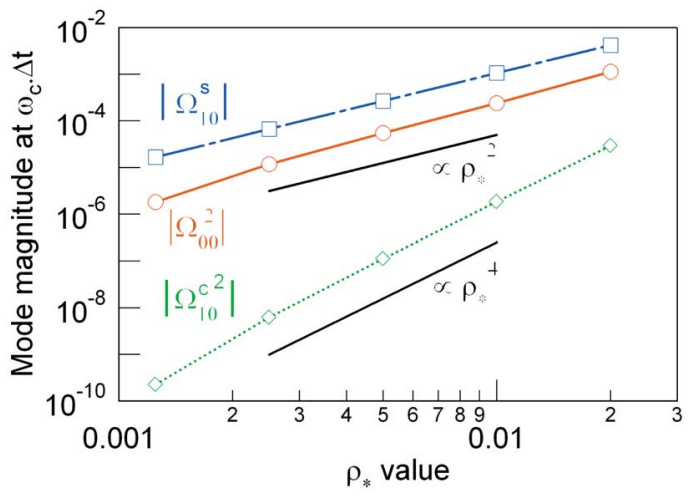

FIG. 2. (Color online) Dependence with $\rho_{\star}$ of the amplitudes of $\phi_{00}, \phi_{10}^{s}$ and $\phi_{10}^{c}$, when initial state is $f_{\mathrm{LM}}$. Values are taken at $\Delta t=15 \omega_{c}^{-1}$.

Those predictions are twofold: (i) on the temporal growth of $\phi_{00}, \phi_{10}^{s}$, and $\phi_{10}^{c}$ and (ii) on their respective amplitudes at the first saving time step $\Delta t$. On the one hand, the slopes which capture the time exponent agree remarkably well until approximately $700 \omega_{c}^{-1}$. At this time, the electric drift and the parallel dynamics are brought into play. The forthcoming Sec. IV will quantitatively discuss this point. On the other hand, the prediction on the amplitudes at $\Delta t=15 \omega_{c}^{-1}$ cannot be straightforwardly compared; a mismatch does exist for numerical reasons between the analytical prediction of the initial amplitudes and their numerical counterparts. Appendix B details the following result: this mismatch remains well below the accessible numerical error bars. Although a direct test of the prediction on the amplitudes $\left|\Omega_{10}^{s}\right|,\left|\Omega_{00}^{2}\right|$, and $\left|\Omega_{10}^{c}{ }^{2}\right|$ in Eqs. (17), (19), and (21) is difficult with the present discretization, it is possible to check their parametric dependencies. Those equations give a strong dependence with $\rho_{\star}$. Equations (17) and (21) predict that

$$
\left|\Omega_{10}^{s}\right|, \quad\left|\Omega_{00}^{2}\right| \propto \rho_{\star}^{2}
$$

and, similarly, Eq. (19) predicts that

$$
\left|\Omega_{10}^{c}{ }^{2}\right| \propto \rho_{\star}^{4} .
$$

Five simulations were run, which were initialized with the local Maxwellian and well below the ITG instability threshold, see Fig. 2 . The only varying parameter was $\rho_{\star}$ in the set, $\rho_{\star}=\{1 / 800,1 / 400,1 / 200,1 / 100,1 / 50\}$. The expected parametric dependencies in Eqs. (23) and (24) are indeed very well recovered numerically.

The physical picture underlying those results can now be drawn. As expected, the first dominant term at short times is the up-down $\phi_{10}^{s}$ dipolar electric field. It is linearly driven in time by the up-down asymmetry of the vertical curvature drift. The cosine component is orders of magnitude smaller. Quasilinear coupling of $\phi_{10}^{s}$ to $f_{00}$ generates via the quasineutrality equation an axisymmetric flow associated with the $\partial_{r r} \phi_{00}$ term. Differently speaking, a poloidal velocity $v_{\text {pol }} \propto d \phi_{10}^{s} / d t \cos \theta$ builds up in time to enforce quasineutrality and is responsible for large scale sheared $\phi_{00}$ flows in the poloidal plane.

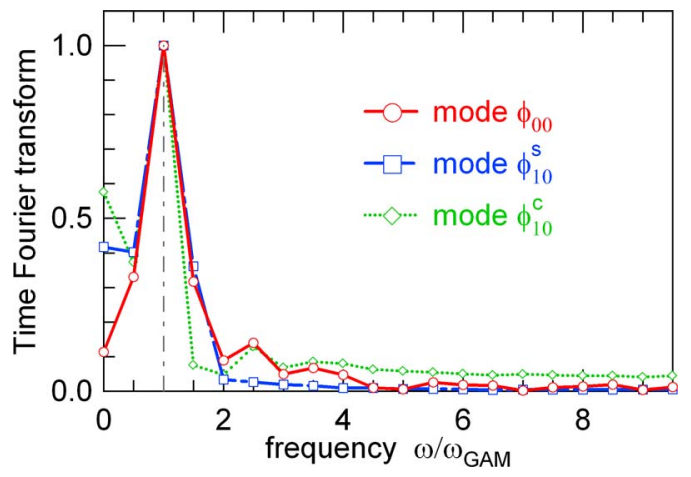

FIG. 3. (Color online) Fourier time spectrum analysis of $\phi_{00}, \phi_{10}^{s}$, and $\phi_{10}^{c}$. Frequencies are normalized to the GAM frequency $\left(\rho_{\star}=1 / 400\right)$.

\section{SATURATION PROCESSES FOR THE POLOIDAL ELECTRIC FIELD}

The strong dependencies with $\rho_{\star}$ contained in Eqs. (17), (19), and (21) do not mean that these flows remain weak. Indeed, an upper boundary of the time growth is given by the time $\tau_{\text {sat }}$ at which either the parallel dynamics or the electric drift velocity are sufficient to counterbalance the charge polarization. The five simulations used for Fig. 2 also show that the saturation level of $\phi_{00}$ (close to $0.17 T_{\mathrm{eq}} / e$ ) remains unchanged for each value of $\rho_{\star}$ in the five runs. Therefore, to remain consistent with Eq. (23), $\tau_{\text {sat }}$ also has to scale with $\rho_{\star}$, $\tau_{\text {sat }} \propto \rho_{\star}^{-1}$. So, in the end, the saturation level of these polarization flows remains significant, whatever the value of $\rho_{\star}$.

Two physical mechanisms can be invoked to keep the radial electric field from growing, respectively associated with the electric drift and the parallel dynamics. It is well known that without collisions, an axisymmetric $\phi_{00}$ flow is nonlinearly damped towards a nonvanishing residual value. ${ }^{11}$ It saturates since nonlinearly coupled with Landau damped $n=1$ sidebands and oscillates at the geodesic acoustic mode $(\mathrm{GAM})$ frequency, ${ }^{12} \omega_{\mathrm{GAM}} \sim \sqrt{7 / 4+\tau} v_{T} / R$. Here, $\tau=T_{e} / T_{i}(=1)$ for adiabatic electrons and $v_{T}$ is the electron thermal velocity. We plot in Fig. 3 the Fourier spectral analysis of $\phi_{10}^{s}, \phi_{00}$, and $\phi_{10}^{c}$. As expected, they are peaked at the $\omega_{\mathrm{GAM}}$ frequency. The width of the peaks comes from the finite time resolution available in this simulation. This piece of physics appears as soon as the electric drift is included in the model, with a characteristic time $\tau_{E}$,

$$
\tau_{E} \sim \omega_{\mathrm{GAM}}^{-1} .
$$

And as expected, we find $\tau_{E} \propto \rho_{\star}^{-1}$. Details of the calculation of $\tau_{E}$ are given in Appendix C. At $\rho_{\star}=1 / 400, \tau_{E} \approx 670 \omega_{c}^{-1}$, which is in good agreement with Fig. 1. At that time, the electric potential starts indeed to depart from the power law predicted by Eqs. (17), (19), and (21).

The second saturation mechanism for the electric potential depends on the parallel dynamics. It consists of building up a parallel flow which will compensate the ever existing polarization due to the curvature drift. No further electric field is then required to satisfy quasineutrality. Appendix D contains the detailed calculation. When introducing the parallel dynamics, Eq. (16) is modified into 


$$
f_{10}^{s}=\frac{v_{d} f_{\mathrm{LM}}}{\mathcal{L}_{p}} t-v_{\|} \frac{\mu B}{T_{\mathrm{eq}}} \frac{r}{q R^{2}} f_{\mathrm{LM}} t .
$$

The first term is due to the inhomogeneity of the magnetic field and triggers the growth of the electric potential already discussed in the previous section. The second term is new, due to the parallel dynamics. Importantly, at short times, it does not modify the dynamics of the electric potential discussed in Sec. III. Indeed, one may recall that in order to calculate $\phi_{10}^{s}$-thus also $\phi_{00}$ and $\phi_{10}^{c}$-the sine component $f_{10}^{s}$ is integrated over phase space. Since the new term in Eq. (26) is odd in $v_{\|}$, it does not contribute to $\phi_{10}^{s}$. Nonetheless, because of this symmetry-breaking term in $v_{\|}$, the distribution function keeps departing from a local Maxwellian. Now, when keeping the second order terms in $\epsilon$, a new term-even in $v_{\|}$-is added to Eq. (26). With this new term detailed in Appendix D, an important new piece of physics is added to the model, allowing for a parallel flow to build up. The back reaction of this flow on the level of generated electric potential can be quantified. It is found that the introduction of the parallel dynamics results in a $t^{3}$ correction term for $\phi_{10}^{s}$ (and a $t^{4}$ correction term for $\phi_{00}$ and $\phi_{10}^{c}$ ),

$$
\frac{e \phi_{10}^{s}}{T_{\mathrm{eq}}} \approx \frac{2 \rho_{s} c_{s}}{R_{0} L_{p}} t-\frac{2 \rho_{s} c_{s}}{R_{0}} \frac{T_{\mathrm{eq}}}{m q^{2} R^{2}}\left(\frac{1}{L_{p}}+\frac{1}{L_{T}}\right) t^{3} .
$$

The quadratic corrections in time for $\phi_{00}$ and $\phi_{10}^{c}$ are straightforwardly calculated from the above expression. The first term describes the growth of the electric potential at short times as discussed in Sec. III. As expected, the negative sign in front of the second term shows that the development of a parallel flow leads to a decrease of this growth. The perturbative analysis of the latter section breaks when the cubic term balances the linear term. It gives an estimate for the characteristic building-up time of the parallel flow,

$$
\tau_{\|} \sim \frac{q R}{v_{T}} \sqrt{1+\frac{L_{p}}{L_{T}}},
$$

where $v_{T}$ is the thermal ion velocity. At $\rho_{\star}=1 / 400$, $\tau_{\|} \approx 2160 \omega_{c}^{-1}$, which is a very good estimate of the saturation time of the flows, as exemplified in Fig. 1. Its necessary parametric dependence with $\rho_{\star}$ is also right since indeed $\tau_{\|} \propto \rho_{\star}^{-1}$.

\section{GENERIC STEADY STATE OF TURBULENCE}

A convenient choice for an equilibrium solution of the gyrokinetic equation is provided by the local Maxwellian in which the radial coordinate $\psi=\int_{0}^{r} r^{\prime} d r^{\prime} / q$ is replaced by an effective coordinate $\bar{\psi}$, function of the motion invariants. ${ }^{2-5}$ This choice is referred to as the canonical Maxwellian,

$$
f_{\mathrm{CM}}=\frac{n(\bar{\psi})}{[2 \pi T(\bar{\psi}) / m]^{3 / 2}} e^{-\mathcal{H}(E, r) / T(\bar{\psi})} e^{+\ell \phi(\bar{\psi}) / T(\bar{\psi})},
$$

where $\mathcal{H}=m v_{\|}^{2} / 2+\mu B+e \phi$ is the total energy. In particular, $\bar{\psi} \equiv P_{\varphi} / e=\psi(r)+m R v_{\varphi} / e$ is a function of $P_{\varphi}$, which explicitly depends on the parallel velocity. The "density" $n$ and "temperature" $T$ in Eq. (29) are no longer radial profiles, but functions of the motion invariant $P_{\varphi}$. Such a class of distri-

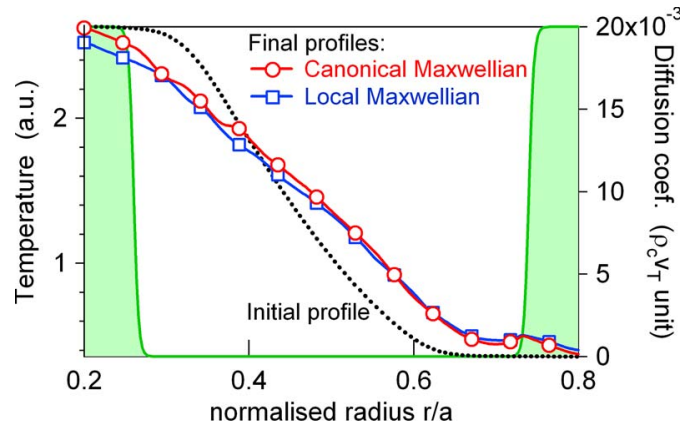

FIG. 4. (Color online) Initial and final temperature profiles when starting from the canonical and the local Maxwellian (initial temperature is the same). The ad hoc radial diffusion coefficient is nonzero in buffer zones only, at $r<0.28 a$ and $r>0.72 a$.

bution functions intrinsically allows for the existence of a parallel flow to counterbalance the polarization drift. In GYSELA, $\bar{\psi}$ is replaced by an effective radial coordinate $\bar{r}$, with the dimension of a length, derived from this third invariant $P_{\varphi}$,

$$
\bar{r}=r_{0}-\frac{q_{0}}{r_{0}}\left[\psi(r)-\psi\left(r_{0}\right)\right]-\frac{m q_{0}}{e B_{0} r_{0}}\left[R v_{\|}-R_{0} \overline{v_{\|}}\right],
$$

where quantities with label " 0 " are defined at half-radius of the simulation box. The last term in Eq. (30) is by definition a motion invariant,

$$
\overline{v_{\|}}=\operatorname{Sign}\left(v_{\|}\right) \sqrt{\frac{2}{m}} \sqrt{E-\mu B_{\max }} \mathcal{Y}\left(E-\mu B_{\max }\right)
$$

with $\mathcal{Y}$ the Heaviside function and $B_{\max }$ the maximum of the magnetic field on the whole simulation box. So as to minimize the parallel flow, $\bar{v}_{\|}$in Eq. (30) is chosen such that $\left(R v_{\|}-R_{0} \bar{v}_{\|}\right)$scales like $\rho^{\star}$. It is important to notice that even though the difference between $r$ and $\bar{r}$ is small, it carries a new piece of physics which intrinsically allows for a parallel flow; the fact that the guiding-centers deviate from a magnetic field line by a few Larmor radii (a banana width at most). The canonical initial state naturally ensures the fluid force balance equation $E_{r}+U_{\theta} B_{\varphi}-U_{\varphi} B_{\theta}=\nabla p /(n e)$. Especially, the canonical initial state gives the correct neoclassical poloidal velocity in the collisionless limit: in the early linear phase, $U_{\theta} \simeq 0.7 \nabla T$ which is very close from the theoretical asymptotic neoclassical value $U_{\theta} \simeq 0.6 \nabla T$ (see Fig. 1 in Ref. 14).

Two simulations at $\rho_{\star}=1 / 200$ and with the same initial temperature gradient $R / L_{T} \approx 12$-well above the thresholdhave been compared up to the nonlinear regime. They only differ by their initial distribution function, either canonical or local Maxwellian. These global simulations used fixed boundary conditions with $\phi=0$ enforced at the radial boundaries, periodic boundary conditions in $(\theta, \varphi)$, and noperturbation conditions in the nonperiodic $r$ and $v_{\|}$directions for the distribution function. Two thermal baths lay at $r \leqslant 0.2 a$ and $r \geqslant 0.8 a$, complemented with two radially localized diffusive regions inside the simulation box (see Fig. 4) in which a source term $S(r)=\partial_{r}\left[D(r) \partial_{r} f\right]$ is added. In these buffer zones, the gyrokinetic equation reads $\mathrm{d} f / \mathrm{d} t=S(r)$. The 


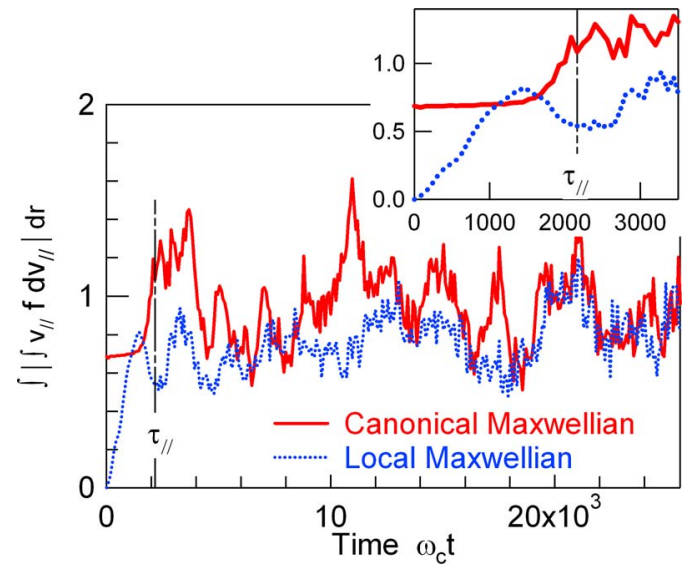

FIG. 5. (Color online) Time evolution of the total amount of parallel flow across the midplane. Note the presence of the absolute value in the definition of the "total amount of parallel flow" within the integral which allows us to include Pfirsch-Schlüter-type flows, which would otherwise vanish by symmetry. The inset is a zoom at early times $\left(\rho_{\star}=1 / 200\right)$.

two diffusive buffer regions at the edge boundary layers are essential to reach a quasisteady state. Indeed, they lead to an effective coupling of the system with the radial boundary conditions where the temperature is prescribed. The effect of the diffusive buffer zones is twofold: (i) they damp the fluctuations towards zero, consistently with the boundary conditions, and (ii) they allow for the diffusive exchange of energy between the thermal baths and the system. It is worth noticing that in such a configuration, the injected energy flux in the system is not constant in time; it is a priori unknown and is an output of the simulation. Alternatively, one could think of simulations at constant (or at least controlled) incoming energy flux. In this case, the temperature freely evolves at one of the radial boundary conditions at least. Such situations would be close to so-called "flux-driven" fluid simulations. $^{13}$

In both nonlinear simulations, we calculated 3200 time steps $\Delta t \approx 5-10 \omega_{c}^{-1}$ on half a torus. The grid mesh is regular and consists of $\sim 2 \times 10^{9}$ grid points in the $5 D$ phase space, more precisely $\left(r, \theta, \varphi, v_{\|}, \mu\right)=(256,256,128,32,8)$. A broad spectrum of low amplitude, random phasing, toroidal and poloidal perturbation modes is added to these initial states. For convenience, we will call the total amount of parallel flow across the midplane (at poloidal angle $\theta=0$ ) the following quantity: $\int \mathrm{d} r\left|\int \mathrm{d} v_{\|} v_{||} f\right|$. Figure 5 plots this quantity as a function of time (the absolute value enables to take into account Pfirsch-Schlüter-type flows whose average over a flux surface would vanish by symmetry). As expected, in the noncanonical case, a parallel flow builds up from nothing on the typical time $\tau_{\|}$, Eq. (28) (inset of Fig. 5). It saturates at the same level as the initial magnitude in the canonical case; both systems end up with a comparable level of parallel flows. The quantity $\int \mathrm{d} r\left|\int \mathrm{d} v_{\|} v_{\|} f\right|$ provides an estimate of the parallel flows, including up-down asymmetric ones.

At shorter times than $\tau_{\|}$, the strong sheared poloidal flows discussed in Sec. III play a dominant role. Their influence on the turbulence is shown in Fig. 6. The time evolution of the heat flux averaged over the central $70 \%$ of the simulation box-corresponding to the width of the global

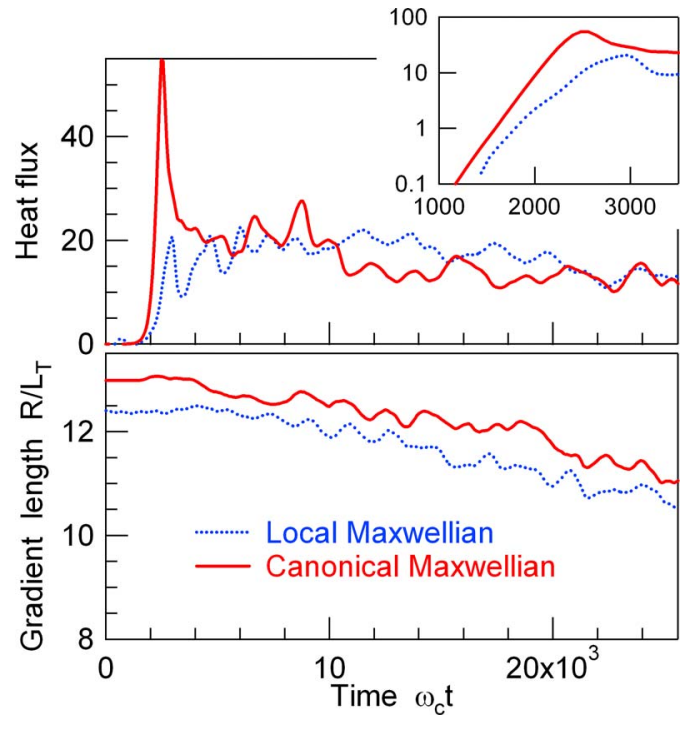

FIG. 6. (Color online) Time evolution of the heat flux and the temperature gradient length in both canonical and noncanonical cases. The inset is a zoom at early times, its $y$-axis is in $\log$ scale $\left(\rho_{\star}=1 / 200\right)$.

mode-is plotted for the two cases. The turbulent transport is delayed when starting from an initial local Maxwellian. As illustrated in the inset of Fig. 6, the shear flow turns out to reduce the effective growth rate, by a factor of about $35 \%$. Such an effect qualitatively agrees with the theoretical predictions, ${ }^{15-17}$ although the reduction appears to be much smaller than expected. The turbulence overshoot at the end of the linear phase is also less pronounced. Note that the system is almost in a statistical steady state, in the sense that the mean temperature gradient relaxes adiabatically as compared to the fluctuations. Indeed, it has relaxed (i) similarly for the two different initial states and (ii) less than $33 \%$ for the canonical case and $34 \%$ for the noncanonical case between 10000 and $25000 \omega_{c}^{-1}$, while the heat flux has remained fairly constant. Nonetheless, the temperature profile keeps on relaxing at longer times, as illustrated in Fig. 6. The incoming energy flux should be maintained in order to avoid such a long-term relaxation in global and full- $f$ simulations.

After the turbulence overshoot (for times larger than 2 to $\left.3 \tau_{\|}\right)$, the heat flux levels for the canonical and noncanonical cases are comparable. This global result does not allow for a straightforward prediction on the saturation levels of the electric potential in both cases. Figure 7 shows that the levels of the axisymmetric flow $\left|\phi_{00}\right|$ and the root mean square $\phi_{\mathrm{RMS}} \equiv \sqrt{\left\langle\delta \phi^{2}\right\rangle_{\theta, \varphi}}$ in the two cases are alike over the whole nonlinear phase. This result is not yet fully understood. Just as well, the statistical properties of the turbulence are almost identical in both cases. Figure 8 compares the time and radial correlation functions calculated in the fully developed turbulent state, since the end of the overshoot (from 5000 to $25000 \omega_{c}^{-1}$ ). The typical size of a turbulent eddy is independent of the initialization and of the order of $6 \rho_{s}$. Similarly, the correlation time is also independent of the initial state and of the order of $800 \omega_{c}^{-1}$.

In the collisionless regime, provided the inevitable profile relaxation is very slow (no flux-driven conditions), after 


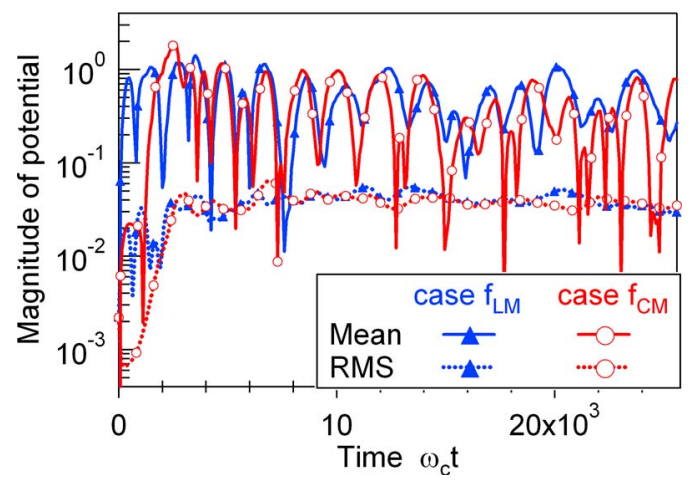

FIG. 7. (Color online) Comparison of the levels of $\phi_{\text {RMS }}$ and of the mean flows $\left|\phi_{00}\right|$ in both the canonical $f_{\mathrm{CM}}$ and noncanonical $f_{\mathrm{LM}}$ cases $\left(\rho_{\star}=1 / 200\right)$.

2 to 3 times $\tau_{\|}$the turbulence develops and washes out very efficiently any possible memory the plasma could retain of its initial state.

\section{TRANSIENT INITIAL STATE MEMORY: DISCUSSION AND CONCLUSION}

Let us start from a linearly unstable initial state in which a small initial perturbation exponentially grows in time. Two generic and very different cases can be considered. First of all, (i) the initial state is a (quasi) stationary state which evolves on a time scale $\tau_{s}$ which is large as compared to the inverse of the linear growth rate $\gamma_{\operatorname{lin}}^{-1}$ of the instability, $\tau_{s} \gamma_{\text {lin }} \gg 1$. This case is exemplified by the choice of the canonical Maxwellian, Eq. (29) as an initial distribution function. Secondly, (ii) the initial state is nonstationary and its characteristic self-organizing time $\tau_{s}$ is comparable to $\gamma_{\text {lin }}^{-1}$, $\tau_{s} \gamma_{\text {lin }} \sim 1$. The local Maxwellian, Eq. (10), is a representative initial state for (ii). In this latter case, to the instability-driven evolution of the plasma superimposes the self-organizing dynamics due to the nonequilibrium initial state. A priori, this can lead to very different dynamics as compared to case (i) in which the evolution of the plasma is only due to the instability.

Transiently indeed, sheared poloidal flows (including zonal flows) are generated in the collisionless regime by the nonstationary local Maxwellian initial state. They develop over the whole simulation box on time scales comparable to the ITG growth rate. Such flows are several orders of magnitude smaller when the initial state is a stationary canonical distribution function. This paper clarifies the underlying physical mechanism: these flows emerge as a result of the vertical plasma polarization which is due to the inhomogeneity of the magnetic field. The poloidally sinusoidal (resp. axisymmetric) component of the electric potential grows linearly (resp. quadratically) in time. Such electric flows saturate due to both the onset of GAMs and the development of parallel flows, analogous to the Pfirsch-Schlüter current. These latter flows efficiently counterbalance the vertical charge polarization. We analytically predict their symmetries, time evolution, initial amplitudes (or at least their parametric dependencies), and saturation time up to the beginning of the nonlinear phase $\left(t<\tau_{\|}\right)$. These results are in very good agree-
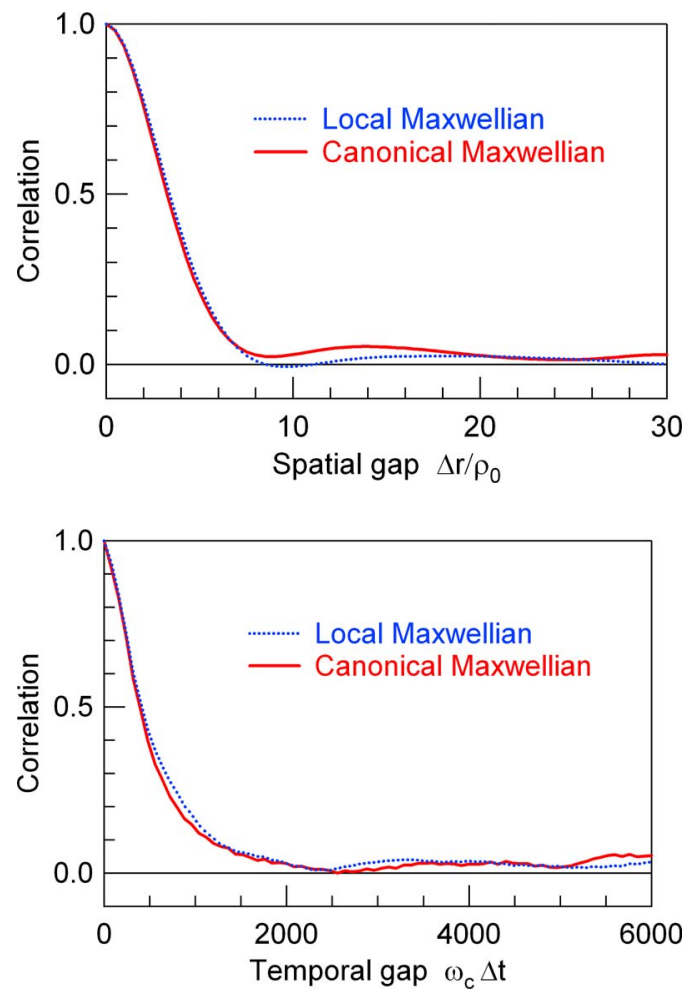

FIG. 8. (Color online) Comparison of the radial and temporal correlation lengths of the electric potential over the whole nonlinear phase in both the canonical and noncanonical cases $\left(\rho_{\star}=1 / 200\right)$.

ment with simulations. In this transient regime, the $\mathbf{E} \times \mathbf{B}$ shearing rate effectively competes with the linear growth rate of the instability and the onset of the turbulence is delayed, as qualitatively already noticed in Refs. 3-5.

At long times though, irrespective of the choice of the initial state, the turbulence robustly develops, with identical statistical properties. This is in marked contrast with the claim of different final states, depending on the choice of the initialization. A new ingredient is necessary to understand this difference. As expected from the ITG context, the mean temperature gradient appears to be that crucial element. In global simulations, the problem consists of its unavoidable relaxation (no flux-driven conditions). In order to minimize it, small diffusive buffer zones at the radial edge of the simulation box have been implemented in GrSELA. They efficiently allow the coupling of the plasma to a free energy reservoir, in our case modelled by two thermal baths. With these conditions, the mean temperature gradient relaxes (i) very slowly and (ii) identically for both the two different considered initial states (see Fig. 6). These results allow us to run simulations during much larger times well above the threshold, which leads to crucial differences as compared to the latter works. Indeed, the more the mean temperature gradient relaxes, the weaker the drive on the turbulent modes. When the system is not coupled to an external source of free energy, as in Refs. $3-5$, this relaxation leads to a marginally stable state on time scales comparable to the linear growth rate. In this case, the turbulent modes are hindered and the onset of the turbulence can be quenched. Conversely, when the system can feed on an external source of free energy, the 
turbulence keeps existing on larger times. In this case, the turbulence is only delayed and its nature is ultimately unaltered; the same level of parallel and axisymmetric flows, the same level of transport and the same correlation times and lengths are obtained (see, respectively, Figs. 5, 7, 6, and 8). The shearing rate due to the axisymmetric flows (including the zonal flows) which develop in the case of a local Maxwellian initial state is not sufficient to quench the onset of the turbulence. Indeed, these flows develop over the whole simulation box on large radial scales, generating a large-scale shear as compared to the typical size of the turbulent modes and the turbulence is ultimately almost unaffected.

As gyrokinetic simulations are numerically heavily demanding, the choice of an initial canonical state in global collisionless simulations is nonetheless important. Indeed, it allows us to address the relevant physics of turbulence growth and nonlinear saturation right from the start, avoiding long transients. In this case, the gain in terms of CPU time can be appreciable. Besides, the local Maxwellian case could prevent the onset of the instability in some specific cases, if the transient polarization electric field turns out to be strong enough to hinder the growth of the unstable modes. This could be all the more critical for those simulations run close to the linear stability threshold. Such a physics is expected to bear some similarities with the so-called Dimits upshift, when the onset of self-generated zonal flows prevents the development of the instability. ${ }^{18}$ In collisional plasmas, the distribution function is forced towards a local Maxwellian and this problem should be less marked. Still, in order to genuinely sustain a steady turbulent state on a confinement time, flux-driven conditions with a prescribed and controlled heat source should be implemented.

\section{ACKNOWLEDGMENTS}

The authors want to thank Ch. Passeron for constant help in the development of the code and to acknowledge many fruitful discussions at the Festival de Théorie in Aix-enProvence, 2007.

\section{APPENDIX A: SHORT-TIME DYNAMICS GOVERNED BY THE CURVATURE AND GRAD-B DRIFTS}

We start with the following equation:

$$
\partial_{t} f+\mathbf{v}_{D} \cdot \nabla f=0
$$

We concentrate on the leading order contributions in $\epsilon$. The latter equation reads

$$
\partial_{t} \delta f_{\mathrm{LM}}=v_{d}\left(\sin \theta \partial_{r} f+\frac{\cos \theta}{r} \partial_{\theta} f\right)
$$

where $v_{d}=\left(m v_{\|}^{2}+\mu B\right) / e R_{0} B_{0}$. Similarly, the quasineutrality equation, Eq. (9), reads

$$
\begin{gathered}
\phi_{10}^{s} \sin \theta+\phi_{10}^{c} \cos \theta-\rho_{s}^{2}\left(\partial_{r r} \phi+\frac{\partial_{\theta \theta} \phi}{r^{2}}\right) \\
=\frac{T_{\mathrm{eq}}}{e n_{\mathrm{eq}}} \frac{2 \pi B}{m} \int_{0}^{\infty} d \mu \int_{-\infty}^{+\infty} d v_{\|} \delta f_{\mathrm{LM}} .
\end{gathered}
$$

For the sake of simplicity, we assume $T_{i}=T_{e}=T_{\mathrm{eq}}$. At leading order in $\epsilon$, the $\sin \theta$ component reads

$$
f_{10}^{s} \approx v_{d} \frac{f_{\mathrm{LM}}}{\mathcal{L}_{p}} t
$$

with $\mathcal{L}_{p}^{-1} \equiv L_{n}^{-1}+L_{T}^{-1}\left(E / T_{\mathrm{eq}}-3 / 2\right) . \quad$ We recall that $L_{X}^{-1}$ $\equiv \partial_{r} X / X$ and $\kappa_{X} \equiv \partial_{r r} X / X$. In particular, $L_{p}$ is the pressure gradient length. Similarly, the $f_{10}^{c}$ component is such that

$$
\partial_{t} f_{10}^{c} \approx \frac{\phi_{10}^{s}}{r B_{0}} \frac{f_{\mathrm{LM}}}{\mathcal{L}_{p}}
$$

At last, the evolution equation for the axisymmetric perturbation $f_{00}$ reads

$$
\begin{aligned}
\partial_{t t} f_{00} \approx & \frac{v_{d}^{2}}{2} \frac{f_{\mathrm{LM}}}{\mathcal{L}_{p}}\left\{\frac{1}{\mathcal{L}_{p}}-\frac{\partial_{r} \mathcal{L}_{p}}{\mathcal{L}_{p}}+\frac{1}{r}\right\} \\
& +\frac{\mu f_{\mathrm{LM}}}{2 R_{0} T_{\mathrm{eq}}} \partial_{t}\left(\partial_{r} \phi_{10}^{s}-\frac{\phi_{10}^{s}}{r}\right) .
\end{aligned}
$$

At this stage, $\phi_{10}^{s}$ remains unknown. The quasineutrality equation then allows us to explicitly solve Eqs. (A5) and (A6). We define $\langle\mathcal{G}\rangle \equiv 2 \pi B / m n_{\text {eq }} \int_{-\infty}^{+\infty} d v_{\|} \int_{0}^{\infty} d \mu \mathcal{G} f_{\mathrm{LM}}$. And thus,

$$
\begin{aligned}
& \langle 1\rangle=1 \quad \text { and } m\left\langle v_{\|}^{2}\right\rangle=B\langle\mu\rangle=T_{\mathrm{eq}}, \\
& \left\langle v_{d}\right\rangle=\frac{2 T_{e q}}{e B_{0} R_{0}} \quad \text { and }\left\langle v_{d}^{2}\right\rangle=\frac{7 T_{\mathrm{eq}}^{2}}{e^{2} B_{0}^{2} R_{0}^{2}} .
\end{aligned}
$$

As a consequence, $\left\langle E / T_{\mathrm{eq}}-3 / 2\right\rangle=0$. Taking the time derivative of Eq. (A3) yields the time evolution of $\phi_{10}^{s}$,

$$
\frac{e}{T_{\mathrm{eq}}} \partial_{t} \phi_{10}^{s} \approx\left\langle\frac{v_{d}}{\mathcal{L}_{p}}\right\rangle=\frac{2 T_{\mathrm{eq}}}{e B_{0} R_{0}} \frac{\partial_{r} p_{\mathrm{eq}}}{p_{\mathrm{eq}}} .
$$

Since the $\rho_{s}^{2}\left(\partial_{r r} \phi_{10}^{s}-\phi_{10}^{s} / r^{2}\right)$ term is a $\rho_{\star}$ correction to $\phi_{10}^{s}$, it has been neglected. At first order in $\rho_{\star}$,

$$
\frac{e}{T_{\mathrm{eq}}} \phi_{10}^{s} \approx \frac{2 \rho_{s} c_{s}}{R_{0} L_{p}} t
$$

with $T_{\mathrm{eq}} / e B_{0}=\rho_{s} c_{s}$ and $c_{s}^{2}=T_{\mathrm{eq}} / m$. Injecting the expression of $\phi_{10}^{s}$, Eq. (A10), into Eqs. (A5) and (A6) leads to

$$
\begin{aligned}
f_{10}^{c} \approx & \frac{f_{\mathrm{LM}}}{r R_{0} L_{p} \mathcal{L}_{p}}\left(\rho_{s} c_{s} t\right)^{2}, \\
f_{00} \approx & \left\{\frac{\mu B_{0}}{T_{\mathrm{eq}}} \frac{f_{\mathrm{LM}}}{2 L_{p}}\left(\frac{\rho_{s} c_{s}}{R_{0}}\right)^{2}\left[\frac{2}{L_{T}}-\frac{1}{L_{p}}+L_{p} \kappa_{p}-\frac{1}{r}\right]\right. \\
& \left.+\frac{v_{d}^{2}}{4} \frac{f_{\mathrm{LM}}}{\mathcal{L}_{p}}\left[\frac{1}{\mathcal{L}_{p}}-\frac{\partial_{r} \mathcal{L}_{p}}{\mathcal{L}_{p}}+\frac{1}{r}\right]\right\} t^{2} .
\end{aligned}
$$

Similarly, one can obtain the evolution equations for $\phi_{10}^{c}$ and $\phi_{00}$ from Eq. (A3), 
TABLE I. Summary of the different sources of imprecision for the estimate of the electric potential, e.g., $\phi_{10}^{s}$ ( $\Delta t$ is the first saving time step).

\begin{tabular}{ll}
\hline \hline Analytical: & $\log \phi_{10}^{s}=\log \Omega_{10}^{s, A}+\log \Delta t$ \\
Numerical: & $\log \phi_{10}^{s}=\log \Omega_{10}^{s, N}+\log \Delta t$ \\
Exact: & $\log \phi_{10}^{s}=\log \Omega_{10}^{s, E}+\log \Delta t$ \\
\hline \hline
\end{tabular}

$$
\begin{aligned}
& \frac{e}{T_{\mathrm{eq}}} \phi_{10}^{c} \approx\left\langle\frac{\rho_{s}^{2} c_{s}^{2} t^{2}}{r R_{0} L_{p} \mathcal{L}_{p}}\right\rangle=\frac{\left(\rho_{s} c_{s}\right)^{2}}{r R_{0} L_{p} L_{n}} t^{2}, \\
& -\rho_{s}^{2} \frac{e}{T_{\mathrm{eq}}} \partial_{r r} \phi_{00} \approx\left\langle\frac{f_{00}}{f_{\mathrm{LM}}}\right\rangle .
\end{aligned}
$$

The latter integral can be estimated as

$$
\begin{aligned}
\frac{e}{T_{\mathrm{eq}}} \partial_{r r} \phi_{00} \approx & -\frac{7}{4}\left[\frac{1}{L_{n}}+\frac{4}{7 L_{p} L_{T}}+\frac{12}{7 L_{p}}\left(\frac{1}{L_{p}}+\frac{1}{r}\right)\right. \\
& \left.+\frac{16}{7} \kappa_{p}-\kappa_{n}\right]\left(\frac{c_{s} t}{R_{0}}\right)^{2} .
\end{aligned}
$$

Equations (A10), (A13), and (A15), respectively, correspond to Eqs. (17), (19), and (21). They are predicted to characterize the dynamics of the system in response to the vertical polarization drift.

\section{APPENDIX B: QUANTITATIVE ANALYSIS OF THE DEPARTURE BETWEEN THE ANALYTICAL PREDICTION AND SIMULATIONS}

Equations (17), (19), and (21) are obtained after integrations over phase space. In GYSELA, these integrals are calculated using cubic splines and have a typical precision $\epsilon^{\text {int }}$ $\approx 10^{-4}$ in our simulations, which can be defined as the relative error between the exact solution of the full problem and the numerical result. To explain it more plainly, let us concentrate on $\Omega_{10}^{s}$, Eq. (18). At least three different values of it exist, written $\Omega_{10}^{s, N}, \Omega_{10}^{s, E}$, and $\Omega_{10}^{s, A}$. The first one is given by the numerical simulation; the second one would be the exact solution of the full problem without any source of numerical imprecision and the last one is the analytical prediction we want to check (summary in Table I). What this appendix is going to show for $\phi_{10}^{s}$ is that $\left|\log \Omega_{10}^{s, N}-\log \Omega_{10}^{s, E}\right| \gg \mid \log \Omega_{10}^{s, A}$ $-\log \Omega_{10}^{s, E} \mid$. The same results are also found for $\phi_{00}$ and $\phi_{10}^{c}$. Though we cannot conclude the amplitudes predicted in Eqs. (17), (19), and (21) are recovered numerically, they remain well inside the accessible numerical error bars. In Eq. (17), the relation

$$
\left\langle\frac{v_{d}}{\mathcal{L}_{p}}\right\rangle=\frac{2 T_{\mathrm{eq}}^{2}}{e^{2} B_{0} R_{0}} \frac{\partial_{r} p_{\mathrm{eq}}}{p_{\mathrm{eq}}}
$$

is exact analytically but only approached on a numerical point of view. When integrating over $v_{\|}$and $\mu$, an error $\epsilon^{s}$ is generated,

$$
\frac{e}{T_{\mathrm{eq}}} \phi_{10}^{s} \approx\left[\frac{2 \rho_{s} c_{s}}{R_{0} L_{p}}+\epsilon^{s}\right] t
$$

This error enters the $f_{10}^{c}$ and $f_{00}$ calculation,

$$
\begin{aligned}
f_{10}^{c} \approx & \frac{f_{\mathrm{LM}} \rho_{s} c_{s}}{r \mathcal{L}_{p}}\left[\frac{\rho_{s} c_{s}}{R_{0} L_{p}}+\frac{\epsilon^{s}}{2}\right] t^{2}, \\
f_{00} \approx & \left\{\frac{v_{d}^{2}}{4} \frac{f_{\mathrm{LM}}}{\mathcal{L}_{p}}\left[\frac{1}{\mathcal{L}_{p}}-\frac{\partial_{r} \mathcal{L}_{p}}{\mathcal{L}_{p}}+\frac{1}{r}\right]\right. \\
& \left.+\frac{\mu f_{\mathrm{LM}}}{4 e R_{0}}\left[\partial_{r}\left(\frac{2 \rho_{s} c_{s}}{R_{0} L_{p}}+\xi^{s}\right)-\left(\frac{2 \rho_{s} c_{s}}{r R_{0} L_{p}}+\frac{\epsilon^{s}}{r}\right)\right]\right\} t^{2} .
\end{aligned}
$$

The integration of the former equation (B3) leads to

$$
\frac{e}{T_{\mathrm{eq}}} \phi_{10}^{c} \approx\left\{\frac{\rho_{s}^{2} c_{s}^{2}}{r R_{0} L_{p}}+\frac{\epsilon^{s} \rho_{s} c_{s}}{2 r}\right\}\left\langle\frac{1}{\mathcal{L}_{p}}\right\rangle t^{2}
$$

Let us introduce $\epsilon^{c}$ the error for $\left\langle 1 / \mathcal{L}_{p}\right\rangle$. The first term of Eq. (B5) can be written, $\left(\rho_{s}^{2} c_{s}^{2} / r R_{0} L_{p} L_{n}+\epsilon^{c}\right) t^{2}$ and the second, $\left(\epsilon^{s} \rho_{s} c_{s} / 2 r L_{n}+\epsilon^{c}\right) t^{2}$. Thus,

$$
\left.\frac{e}{T_{\mathrm{eq}}} \phi_{10}^{c} \approx \phi_{10}^{c}\right|_{\epsilon^{s}=0}+\left[2 \epsilon^{c}+\frac{\epsilon^{s} \rho_{s} c_{s}}{2 r L_{n}}\right] t^{2} \text {. }
$$

Proceeding the same way for $\phi_{00}$,

$$
-\rho_{s}^{2} \partial_{r r} \phi_{00} \approx \frac{T_{\mathrm{eq}}}{e n_{\mathrm{eq}}} \frac{2 \pi B}{m} \iint d \mu d v_{\|} f_{00} .
$$

Let us define $f_{00}^{\epsilon^{s}=0}$ given by Eq. (B4) when $\boldsymbol{\epsilon}^{s}=0$. Let us introduce $\epsilon_{1}^{0}$ and $\epsilon_{2}^{0}$ which represent the errors for, respectively, $\left\langle f_{00}^{\epsilon^{s}=0} / f_{\mathrm{LM}}\right\rangle$ and $\langle\mu\rangle$. Thus, Eq. (B7) reads

$$
\left.\frac{e}{T_{\mathrm{eq}}} \partial_{r r} \phi_{00} \approx \frac{e}{T_{\mathrm{eq}}} \partial_{r r} \phi_{00}\right|_{\epsilon^{s}=0}-\frac{1}{\rho_{s}^{2}}\left[\epsilon_{1}^{0}+\epsilon_{2}^{0}-\frac{\epsilon^{s} \rho_{s} c_{s}}{4 r R_{0}}\right] t^{2} .
$$

The smaller the $\rho_{\star}$ parameter, the more important those errors. At $\rho_{\star}=1 / 400$, the mismatch between analytics and numerics is most penalizing,

$$
\begin{aligned}
& \epsilon^{s} \approx-2.78 \times 10^{-6}, \\
& 2 \epsilon^{c}+\frac{\epsilon^{s} \rho_{s} c_{s}}{2 r L_{n}} \approx-2.24 \times 10^{-10}, \\
& \left(\epsilon_{1}^{0}+\epsilon_{2}^{0}\right)\left[\frac{r}{a}\right]^{2}-\frac{\epsilon^{s} \rho_{s} c_{s}(r \log r-r)}{4 a^{2} R_{0}} \approx 8.52 \times 10^{-8} \rho_{\star}^{2} .
\end{aligned}
$$

And thus,

$$
\begin{aligned}
& \epsilon^{s} \approx-2.78 \times 10^{-6}, \\
& \epsilon^{c} \approx-1.09 \times 10^{-10}, \\
& \epsilon_{1}^{0}+\epsilon_{2}^{0} \approx 1.16 \times 10^{-13} .
\end{aligned}
$$

Yet all three errors, Eqs. (B12)-(B14), are well below $\epsilon^{\text {int }}$. 


\section{APPENDIX C: ONSET OF GAMS WITH QUALITATIVE AGREEMENT ON THE FREQUENCY}

We calculate the modification of the prediction on the components of the electric potential Eqs. (17), (19), and (21) when the electric drift is taken into account. We find an oscillating modulation, close to the GAM frequency, as expected from the Rosenbluth and Hinton theory. ${ }^{11}$ Let us consider

$$
\partial_{t} f+\left(\mathbf{v}_{D}+\mathbf{v}_{E}\right) \cdot \nabla f=0 .
$$

We will concentrate on the first order terms in $\epsilon$ since Sec. III already discussed the leading order at short times. Using Eqs. (11)-(13), Eq. (C1) reads

$$
\begin{aligned}
\partial_{t} \delta f_{\mathrm{LM}} \approx & v_{d}\left\{\sin \theta \partial_{r} \delta f_{\mathrm{LM}}+\frac{\cos \theta}{r} \partial_{\theta} \delta f_{\mathrm{LM}}\right\} \\
& +\frac{\mu f_{\mathrm{LM}}}{R T_{\mathrm{eq}}}\left\{\sin \theta \partial_{r} \phi-\frac{\cos \theta}{r} \partial_{\theta} \phi\right\}+\frac{\partial_{\theta} \phi}{r B \mathcal{L}_{p}} f_{\mathrm{LM}} .
\end{aligned}
$$

In the following, we will neglect the terms with a $\cos \theta$ dependence as compared to those in $\sin \theta$. Proceeding the same way than in Sec. III, the first Fourier modes of the distribution function read

$$
\begin{aligned}
& \partial_{t} f_{00} \approx \frac{v_{d}}{2} f_{10}^{s}{ }^{\prime}+\frac{\mu f_{\mathrm{LM}}}{2 R T_{\mathrm{eq}}} \phi_{10}^{s}, \\
& \partial_{t} f_{10}^{s} \approx v_{d} f_{00}^{\prime}+\frac{\mu f_{\mathrm{LM}}}{R T_{\mathrm{eq}}} \phi_{00}^{\prime}, \\
& \partial_{t} f_{10}^{c} \approx \frac{f_{\mathrm{LM}}}{r B \mathcal{L}_{p}} \phi_{10}^{s},
\end{aligned}
$$

where $\phi_{10}^{s}{ }^{\prime} \equiv \partial_{r} \phi_{10}^{s}$. Equations (C3) and (C4) can be recast into

$$
\begin{aligned}
& \partial_{t t} f_{00} \approx \frac{v_{d}^{2}}{2} f_{00}^{\prime \prime}+\frac{\mu f_{\mathrm{LM}}}{R T_{\mathrm{eq}}}\left(\phi_{00}^{\prime \prime}+\frac{\partial_{t} \phi_{10}^{s}}{2}\right), \\
& \partial_{t t} f_{10}^{s} \approx \frac{v_{d}^{2}}{2} f_{10}^{s \prime \prime}+\frac{\mu f_{\mathrm{LM}}}{R T_{\mathrm{eq}}}\left(\frac{\phi_{10}^{s}}{2}+\partial_{t} \phi_{00}^{\prime}\right) .
\end{aligned}
$$

When coupled to the quasineutrality equation, the first two terms in Eqs. (C6) and (C7) due to the curvature drift lead to high-order terms $\propto \partial_{r}^{4} \phi_{00}\left(\right.$ resp. $\left.\partial_{r}^{2} \phi_{10}^{s}\right)$ for $\phi_{00}\left(\right.$ resp. $\left.\phi_{10}^{s}\right)$ which are negligible. The electric potential reads

$$
\begin{aligned}
& \partial_{t t}\left(-\rho_{s}^{2} \frac{e \phi_{00}^{\prime \prime}}{T_{\mathrm{eq}}}\right) \approx \frac{1}{R_{0} B_{0}}\left(\phi_{00}^{\prime \prime}+\frac{\partial_{t} \phi_{10}^{s}}{2}\right), \\
& \partial_{t t}\left(\frac{e \phi_{10}^{s}}{T_{\mathrm{eq}}}\right) \approx \frac{1}{R_{0} B_{0}}\left(\frac{\phi_{10}^{s}}{2}+\partial_{t} \phi_{00}^{\prime}\right), \\
& \partial_{t}\left(\frac{e \phi_{10}^{c}}{T_{\mathrm{eq}}}\right) \approx \frac{\phi_{10}^{s}}{r B}\left\langle\frac{1}{\mathcal{L}_{p}}\right\rangle=\frac{\phi_{10}^{s}}{r B L_{n}},
\end{aligned}
$$

where we made use of Eq. (A7). Since we are interested in times when $\phi_{00}$ or $\phi_{10}^{s}$ start to saturate, the $\partial_{t} \phi_{10}^{s}{ }^{\prime} / 2$ term in
Eq. (C8) is neglected as compared to $\phi_{00}^{\prime \prime}$. This equation has a time-oscillating solution close to the GAM frequency,

$$
\phi_{00}^{\prime \prime} \propto \cos \left(\frac{c_{s}}{R} t+\aleph\right)
$$

with $\aleph$ an arbitrary phase. Given Eq. (C11), a function $\propto \sin \left(c_{s} t / R+\aleph\right)$ is a solution of Eq. (C9). Then, Eq. (C10) leads to $\phi_{10}^{c} \propto \phi_{00}^{\prime \prime}$. All three components of the electric potential have a time-oscillating behavior close to the GAM frequency, as found indeed in Fig. 3. It also clearly scales with $\rho_{\star}$ since $c_{s} / R \propto \rho_{\star}$.

\section{APPENDIX D: PARALLEL FLOWS, A ROBUST SATURATION MECHANISM FOR THE CHARGE POLARIZATION}

With the parallel dynamics, Eq. (15) reads

$$
\partial_{t} f+\mathbf{v}_{D} \cdot \nabla f+v_{\|} \nabla_{\|} f+\frac{\mathrm{d} v_{\|}}{\mathrm{d} t} \partial_{v_{\|}} f=0 .
$$

The sine component equation has the simple form

$$
\partial_{t} f_{10}^{s}=\frac{v_{d} f_{\mathrm{LM}}}{\mathcal{L}_{p}}+\frac{\mu}{m} \nabla_{\|} B \partial_{v_{\|}} f_{\mathrm{LM}}+\frac{v_{\|}}{q R} f_{10}^{c} .
$$

At leading order in $\epsilon$, it can be integrated over time,

$$
f_{10}^{s}=\frac{v_{d} f_{\mathrm{LM}}}{\mathcal{L}_{p}} t-v_{\|} \frac{\mu B}{T_{\mathrm{eq}}} \frac{r}{q R^{2}} f_{\mathrm{LM}} t .
$$

The cosine component equation can also be estimated. To do so and include the contribution of the parallel dynamics, we assume it is initially a perturbation. Equation (A5) is modified into

$$
\partial_{t} f_{10}^{c}=\frac{f_{\mathrm{LM}}}{r B_{0} \mathcal{L}_{p}} \phi_{10}^{s}-\frac{v_{\|}}{q R} f_{10}^{s}
$$

with $\phi_{10}^{s}$ and $f_{10}^{s}$ given, respectively, by Eqs. (17) and (D3). The odd part in $v_{\|}$of $f_{10}^{c}$, written as $\mathcal{O}\left(f_{10}^{c}\right)$, reads

$$
\mathcal{O}\left(f_{10}^{c}\right)=\frac{v_{\|}}{q R} \frac{v_{d} f_{\mathrm{LM}}}{\mathcal{L}_{p}} t .
$$

It allows us to determine $\phi_{10}^{s}$,

$$
\begin{aligned}
\partial_{t}\left(\frac{e \phi_{10}^{s}}{T_{\mathrm{eq}}}\right) & =\left\langle\frac{v_{d}}{\mathcal{L}_{p}}+\frac{\mu}{m} \nabla_{\|} B \frac{\partial_{v_{\|}} f_{\mathrm{LM}}}{f_{\mathrm{LM}}}+\frac{v_{\|}}{q R} \frac{f_{10}^{c}}{f_{\mathrm{LM}}}\right\rangle \\
& =\left\langle\frac{v_{d}}{\mathcal{L}_{p}}\right\rangle-\frac{1}{2}\left\langle\frac{v_{\|}^{2} v_{d}}{\mathcal{L}_{p}}\right\rangle\left(\frac{t}{q R}\right)^{2} .
\end{aligned}
$$

After a little algebra,

$$
\frac{e \phi_{10}^{s}}{T_{\mathrm{eq}}}=\frac{2 \rho_{s} c_{s}}{R_{0} L_{p}} t-\frac{2 \rho_{s} c_{s}}{R_{0}} \frac{T_{\mathrm{eq}}}{m q^{2} R^{2}}\left(\frac{1}{L_{p}}+\frac{1}{L_{T}}\right) t^{3} .
$$

The introduction of the parallel dynamics results in a $t^{3}$ correction term, which is negligible at short times. It becomes significant when the linear term in this equation is balanced by the cubic term. An upper boundary for this time is 


$$
\tau_{\|} \sim \frac{q R}{v_{T}} \sqrt{1+\frac{L_{p}}{L_{T}}},
$$

where $v_{T}$ is the thermal velocity of the ions. Since the $\phi_{00}$ and $\phi_{10}^{c}$ components of the electric potential are evaluated from $\phi_{10}^{s}$, the parallel dynamics introduce a $t^{4}$-correction to their $t^{2}$-dependence. Also, $\tau_{\|}$clearly scales with $\rho_{\star}$ since $R / v_{T} \propto \rho_{\star}^{-1}$.

${ }^{1}$ P. H. Diamond, S.-I. Itoh, K. Itoh, and T. S. Hahm, Plasma Phys. Controlled Fusion 47, R35 (2005).

${ }^{2}$ Y. Idomura, S. Tokuda, and Y. Kishimoto, Nucl. Fusion 43, 234 (2003).

${ }^{3}$ P. Angelino, A. Bottino, R. Hatzky, S. Jolliet, O. Sauter, T. M. Tran, and L. Villard, Phys. Plasmas 13, 052304 (2006).

${ }^{4}$ X. Garbet, Y. Sarazin, V. Grandgirard, G. Dif-Pradalier, G. Darmet, Ph. Ghendrih, P. Angelino, P. Bertrand, N. Besse, E. Gravier, P. Morel, E. Sonnendrücker, N. Crouseilles, J.-M. Dischler, G. Latu, E. Violard, M. Brunetti, S. Brunner, X. Lapillonne, T.-M. Tran, L. Villard, and M. Boulet, Nucl. Fusion 47, 1206 (2007).

${ }^{5}$ G. Dif-Pradalier, V. Grandgirard, Y. Sarazin, X. Garbet, and P. Ghendrih, Commun. Nonlinear Sci. Numer. Simul. 13, 65 (2008).

${ }^{6}$ V. Grandgirard, M. Brunetti, P. Bertrand, N. Besse, X. Garbet, P. Ghendrih, G. Manfredi, Y. Sarazin, O. Sauter, E. Sonnendrucker, J. Vacla- vik, and L. Villard, J. Comput. Phys. 217, 395 (2006).

${ }^{7}$ V. Grandgirard, Y. Sarazin, P. Angelino, A. Bottino, N. Crouseilles, G. Darmet, G. Dif-Pradalier, X. Garbet, Ph. Ghendrih, S. Jolliet, G. Latu, E. Sonnendrücker, and L. Villard, Plasma Phys. Controlled Fusion 49, B173 (2007).

${ }^{8}$ Y. Sarazin, V. Grandgirard, E. Fleurence, X. Garbet, P. Ghendrih, P. Bertrand, and G. Depret, Plasma Phys. Controlled Fusion 47, 1817 (2005).

${ }^{9}$ A. Brizard and T. Hahm, Rev. Mod. Phys. 79, 421 (2007).

${ }^{10}$ D. H. E. Dubin, J. A. Krommes, C. Oberman, and W. W. Lee, Phys. Fluids 26, 3524 (1983).

${ }^{11}$ M. N. Rosenbluth and F. L. Hinton, Phys. Rev. Lett. 80, 724 (1998).

${ }^{12}$ H. Sugama and T.-H. Watanabe, Phys. Plasmas 13, 012501 (2006).

${ }^{13}$ X. Garbet, Y. Sarazin, P. Beyer, P. Ghendrih, R. Waltz, M. Ottaviani, and S. Benkadda, Nucl. Fusion 39, 2063 (1999).

${ }^{14}$ Y. B. Kim, P. H. Diamond, and R. J. Groebner, Phys. Fluids B 3, 2050 (1991).

${ }^{15}$ H. Biglari, P. H. Diamond, and P. W. Terry, Phys. Fluids B 2, 1 (1990).

${ }^{16}$ R. E. Waltz and G. D. Kerbel, Phys. Plasmas 2, 2408 (1995).

${ }^{17}$ T. S. Hahm and K. H. Burrell, Phys. Plasmas 3, 427 (1996).

${ }^{18}$ A. M. Dimits, G. Bateman, M. A. Beer, B. I. Cohen, W. Dorland, G. W. Hammett, C. Kim, J. E. Kinsey, M. Kotschenreuther, A. H. Kritz, L. L. Lao, J. Mandrekas, W. M. Nevins, S. E. Parker, A. J. Redd, D. E. Shumaker, R. Sydora, and J. Weiland, Phys. Plasmas 7, 969 (2000). 